\title{
HIV co-infection with tuberculous and non-tuberculous mycobacteria in western Kenya: challenges in the diagnosis and management
}

Nyamogoba H DN ${ }^{1,2, *}$, Mbuthia $G^{3}$, Mining $S^{1}$, Kikuvi $G^{2}$, Biegon $R^{1}$, Mpoke $S^{4}$, Menya $D^{3}$, Waiyaki P G ${ }^{4}$

1. Moi University School of Medicine, Kenya

2. Institute of Tropical Medicine and Infectious Diseases, Jomo Kenyatta University of Agriculture and Technology, Kenya

3. Moi University School of Public Health, Kenya

4. Kenya Medical Research Institute, Kenya

\begin{abstract}
Background: Tuberculosis (TB) and HIV co-infections have a global prevalence with devastating morbidity and massive mortality, Sub-Saharan Africa being the worst hit.

Objectives: To evaluate the prevalence of TB-HIV co-infection and demonstrate the confusion caused by NTM and HIV/ AIDS co-infection in TB diagnosis and treatment in western Kenya.

Methods: In a cross-sectional study carried out at 10 hospitals in western Kenya, sputa from consenting 872 TB suspects underwent microscopy, and culture on Lowenstein-Jensen and Mycobacteria Growth Index Tube media. Isolates were identified using the Hain's GenoType ${ }^{\circledR}$ Mycobacterium CM and GenoType ${ }^{\circledR}$ Mycobacterium AS kits. A total of 695 participants were screened for HIV using Uni-Gold ${ }^{\mathrm{TM}}$ test and positives confirmed with the enzyme linked immunosorbent assay.

Results: A total of $346(39.7 \%)$ participants were diagnosed with TB. Out of the 346 TB cases, $263(76 \%)$ were tested for HIV infection and $110(41.8 \%$ ) of these were sero-positive (co-infected). The female to male TB-HIV co-infection prevalence ratio (PR) was 1.35. This study reports isolation of non-tuberculous mycobacteria from TB suspects at a rate of $1.7 \%$.

Conclusion: A high TB-HIV co-infection rate was observed in this study. The NTM disease could be misdiagnosed and treated as TB in western Kenya.
\end{abstract}

Key words: Tuberculosis, HIV co-infection, high prevalence, TB diagnosis

African Health Sciences 2012; 12(3):305 - 311 http://dx.doi.org/10.4314/ahs.v12i3.9

\section{Introduction}

Since early 1990s, the HIV/AIDS pandemic has transformed the TB infections to epidemic proportions. Sub-Saharan Africa is the worst hit region in terms of the impact of TB-HIV, where one-third of the estimated 40 million people living with HIV/AIDS are co-infected with $\mathrm{TB}^{1,2}$. While HIV negative persons with latent TB have a $10 \%$ lifetime risk of developing active TB, those with HIV and TB co-infection have a $50 \%$ lifetime risk of developing active TB in a given year ${ }^{3-5}$ fuelling an upsurge in the TB epidemic with an estimated annual mortality of 2 million people worldwide ${ }^{5}$. Mortality

\begin{tabular}{l}
\hline *Corresponding author: \\
Nyamogoba H DN \\
Institute of Tropical Medicine and Infectious \\
Diseases \\
Jomo Kenyatta University of Agriculture and \\
Technology \\
P.O. Box 4606 \\
Eldoret Kenya \\
E-mail: henrynyamogoba@yahoo.co.uk \\
\hline
\end{tabular}

African Health Sciences Vol 12 No 3 September 2012 is reported to increase up to 4-fold in HIV-infected TB patients ${ }^{6}$.

The HIV infection is currently the greatest risk factor for new TB infection, and a potential risk for recurrence of $\mathrm{TB}^{7-9}$. Development of active $\mathrm{TB}$ in HIV infected persons appears to be associated with increased HIV replication which is probably initiated by enhanced cytokine expression ${ }^{10}$ and is responsible for shortened survival rate in HIV infected persons ${ }^{11,12}$. In turn, TB is the leading cause of respiratory morbidity ${ }^{13}$ and mortality in HIVinfected persons globally, and accounts for $44 \%$ of all AIDS-related deaths annually ${ }^{14}$. The TB-HIV coinfection is not only a concern for adults. In South Africa, nearly $25 \%$ of children with HIV in one study developed TB during the course of a year ${ }^{4}$. Patients with HIV and drug-resistant TB have higher risks of adverse drug reactions and drug toxicity, thus requiring increased monitoring by healthcare providers $^{15}$. It is recommended that TB-HIV programmes be well-established before adopting drug-resistant TB-HIV interventions ${ }^{15}$. 
The HIV/AIDS has profound impact on the TB epidemic in Kenya, where up to $60 \%$ of TB patients are feared to be HIV co-infected and the mortality rate attributed to TB in this group being above 130 per $100,000^{16}$. Currently approximately 250 Kenyans die every day from HIV/AIDS-related illnesses ${ }^{17}$. The TB-HIV/AIDS problem is compounded by the emergence of non-tuberculous mycobacteria (NTM) as opportunistic infections in the HIV/AIDS patients, and their treatment is not directly analogous to that of $\mathrm{TB}^{18,19}$. In the present study, the TB-HIV co-infection rate is evaluated after three decades of intervention measures, and the confusion caused by NTM and HIV/AIDS coinfection in TB diagnosis and treatment in western Kenya is demonstrated.

\section{Methods}

\section{Study design}

A cross-sectional study was conducted between September 2007 and September 2009.

\section{Study setting}

The study was conducted at one provincial and nine district hospitals in western Kenya. These were Busia, Bungoma, Kisumu, Migori, Kisii, Narok, Kericho, Uasin Gishu and Lodwar district hospitals, and Nakuru Provincial General Hospital. Western Kenya includes the former Rift Valley, Nyanza and Western Provinces, with a cumulative population of about 19.8 million people. This constitutes about $52.1 \%$ of the Kenyan population, according to the Kenya Census of 2009.

\section{Sampling frame and patient characteristics}

Participants suspected of having pulmonary TB were enrolled into the study between September 2007 and September 2009 as they sought healthcare services at the chest and paediatric clinics. They had to be resident in western Kenya for at least six months prior to the date of enrolment and consented to participate in the study. Cases that had prior TB treatment were excluded. Participants were suspected of having TB if they had a cough of more than two weeks duration and were not responding to antibiotic treatment ${ }^{20}$.

\section{Collection of demographic data}

A questionnaire was used to obtain participant demographic data. Data collected included age, gender, HIV status, and antiretroviral therapy (ART).

\section{Collection of samples}

At least $2 \mathrm{ml}$ of three sputum specimens (spot, early morning, spot) ${ }^{19}$ were collected from 872 participants with suspected TB under the supervision of trained and competent medical staff. The suspects were requested to cough so that expectoration would come from as deep down the chest as possible, and spit into a sterile $50 \mathrm{ml}$ blue cap tubes. The samples were refrigerated at $4^{\circ} \mathrm{C}$ awaiting transportation in cool boxes to the Mycobacteria Reference Laboratory, Moi University School of Medicine (MRL, MUSOM) weekly for analysis. At the MRL, MUSOM, the samples were refrigerated at $4{ }^{\circ} \mathrm{C}$ till processing. The samples were processed within 7 days of collection in order to minimize loss of viability of the mycobacteria. Consenting 695 participants also underwent phlebotomy for HIV testing. The blood was delivered into Vacutainer Brand STERILE interior EDTA (K3) tubes and stored at $-20^{\circ} \mathrm{C}$ awaiting processing. The samples were transported in cool boxes to MRL, MUSOM, Eldoret, and processed within two weeks. The safety for research assistants and healthcare workers during collection and handling of sputum specimens was ensured by observing the WHO guidelines ${ }^{21}$.

\section{HIV testing}

Whole blood obtained from the consenting $695 \mathrm{~TB}$ suspects was allowed to clot. The serum was screened for anti-HIV antibodies using Trinity Biotech Uni-Gold ${ }^{\mathrm{TM}}$ rapid test and positives confirmed with the enzyme linked immunosorbent assay (ELISA), following manufacturers' instructions.

\section{Microscopic examination of specimens}

Microscopic examination for Mycobacterium infection was done after staining specimens with carbol-fuchsin using the $\mathrm{ZN}$ method ${ }^{22}$. A TB suspect was considered to be $\mathrm{ZN}$ smear positive if at least one of the three specimens showed pink/red rod shaped bacteria on microscopy.

\section{Isolation and identification of mycobacteria}

Three sputum specimens from each patient were processed for isolation of mycobacteria following standard protocols ${ }^{23}$. A participant with at least one positive MGIT culture (Culture on LJ was used as a back-up) was considered as a TB case, while those with three negative culture results were regarded as not having TB. The mycobacterial isolates were identified as $M$. tuberculosis complex or species of NTM using Hain’s GenoType ${ }^{\circledR}$ Mycobacterium CM 
and GenoType ${ }^{\circledR}$ Mycobacterium AS Molecular Genetic Assays, following manufacturer's instructions.

\section{Data analysis}

Data was entered in MS Excel 8.0 and analysed using Epi Info version 3.5.1. Descriptive statistics were used to summarize data and proportions compared using Chi-square testing. Odds rations (OR) with 95\% confidence intervals (CI) were calculated to assess age as risk factor with regard to TB-HIV co-infection. $P$-value $<0.05$ was considered as statistically significant.

\section{Ethical issues}

The proposal for this study was approved by the Institute of Tropical Medicine and Infectious Diseases (ITROMID) of Jomo Kenyatta University of Agriculture and Technology and the Kenya Medical Research Institute (KEMRI)'s Scientific Steering Committee (SSC) and Ethical Review Committee (ERC) [SSC No. 837]. It was also approved by Moi University School of Medicine (MU-SOM) / Moi Teaching and Referral Hospital (MTRH) Institutional Research and Ethics Committee (IREC) [FAN No.00092]. Permission was also obtained from respective district health authorities and hospital administrations. Informed consent and/or assent were obtained from candidates or their guardians before they were enrolled into the study. The purpose of the study was explained to the candidates in English, Kiswahili or a local language before written consent and / or assent was sought. Code numbers were used to identify candidates in order to maintain confidentiality. The study did not expose candidates to any unusual risks as competent hospital staff obtained sputum and blood specimens from candidates using standard procedures.

\section{Results}

\section{Study population}

A total of the 872 clients were enrolled into the study, $54.9 \%$ (477) being males and $45.1 \%$ (393) females. Their mean and median ages were 35 and 32 years, respectively. The majority $(33.1 \%)$ were in the $25-$ 34 years age bracket, followed by those in the 35-44 $(21.8 \%)$ and $15-24(18.7 \%)$ age brackets respectively. Paediatric cases (0-14 year age-group) were the lowest with $4.5 \%$, with children 5 years and below accounting for only $0.6 \%$ (table 1 ).
Table 1: Study participants and gender-age distribution

\begin{tabular}{llll}
\hline $\begin{array}{l}\text { Age-group } \\
\text { (years) }\end{array}$ & N (\%) & Males (\%) & Females (\%) \\
\hline $0-14$ & $39(4.5)$ & $22(2.5)$ & $18(2.1)$ \\
$15-24$ & $163(18.7)$ & $80(9.2)$ & $83(9.5)$ \\
$25-34$ & $288(33.1)$ & $162(18.6)$ & $126(14.4)$ \\
$35-44$ & $190(21.8)$ & $108(12.4)$ & $82(9.4)$ \\
$45-54$ & $89(10.2)$ & $108(12.4)$ & $36(4.1)$ \\
$55-64$ & $54(6.2)$ & $29(3.3)$ & $25(2.9)$ \\
$>64$ & $48(5.5)$ & $25(2.9)$ & $23(2.6)$ \\
Total & $\mathbf{8 7 2 ( 1 0 0 )}$ & $\mathbf{4 7 9 ( 5 4 . 9 )}$ & $\mathbf{3 9 3 ( 4 5 . 1 )}$ \\
\hline
\end{tabular}

\section{Mycobacterial infection}

A total of $361(45.1 \%)$ participants were diagnosed with mycobacterial infection. Out of these, $95.8 \%$ $(346 / 361)$ were TB, and 4.2\% (15/361) NTM infections. The overall prevalence of NTM infection was $1.7 \%(15 / 872)$. Up to $6.4 \%(22 / 346)$ of the mycobacterial infection (20/22; $90.9 \% \mathrm{~TB}$ and $2 /$ 22; 9.1 NTM) cases were $\mathrm{ZN}$ smear negative but culture positive. Of the 15 NTM isolates, 5 were identified to species level; $3(20 \%)$ as $M$. intracellulare, and $1(6.7 \%)$ as $M$. fortuitum and $1(6.7 \%)$ as $M$. peregrinum. The remaining 10/15 (66.7\%) NTM isolates could not be identified to species level. A total of $44.3 \%(160 / 361)$ symptomatic cases were $\mathrm{ZN}$ smear positive but culture negative, and were treated for TB. No cultures yielded tuberculous and non-tuberculous mycobacteria co-infection.

\section{HIV infection and ART}

The HIV testing was accepted by 695 participants and $39.1 \%(272 / 695)$ were sero-positive. Forty six $(16.9 \%)$ of those diagnosed with HIV infection were on ART.

\section{Mycobacteria-HIV co-infection}

Out of the 361 mycobacteria infection cases (TB and NTM), $75.9 \%(274 / 361)$ accepted to be tested for HIV infection and $42.7 \%(117 / 274)$ of these were co-infected (table 2). Only $24.1 \%(87 / 361)$ of the TB cases declined to be tested for HIV infection and therefore their status was unknown. 
Table 2: Mycobacteria-HIV co-infection

\begin{tabular}{lcr}
\hline Mycobacteria and HIV status & $\begin{array}{c}\text { Number of } \\
\text { cases }\end{array}$ & \% \\
\hline Mycobacterium- with HIV & 117 & 41.8 \\
co-infection & & \\
Mycobacterium without HIV & 157 & 57.3 \\
Totals & $\mathbf{2 7 4}$ & $\mathbf{1 0 0 \%}$ \\
\hline
\end{tabular}

TB-HIV co-infection

A total of 263 (165 males and 98 females) of the 346 TB cases were among those tested for HIV infection and $41.8 \%(110 / 263)$ were co-infected. Males constituted 55.5\% (61/110) and females $44.5 \%$ (49/110). The female to male TB-HIV coinfection prevalence ratio $(\mathrm{PR})$ was 1.35 . The majority $(40.9 \%)$ of the TB-HIV cases were in the 25-34 year age bracket followed by the 35-44 (24.5\%) and 15$24(19.1 \%)$ year age brackets. However, there was no significant difference in TB-HIV co-infection between the various age-groups with the 0-14 agegroup as the referent exposure (table 3 ).

Table 3: Assessment of age-group as risk factor for TB-HIV co-infection

\begin{tabular}{llllr}
\hline Age-group & $\mathbf{N ~ ( \% )}$ & $\mathbf{O R}$ & $\mathbf{9 5} \% \mathbf{C I}$ & $\boldsymbol{P}$-value \\
\hline $0-14$ & $27(3.9)$ & - & - & - \\
$15-24$ & $127(18.3)$ & 1.14 & $0.36-3.63$ & 0.82 \\
$25-34$ & $236(34.0)$ & 1.36 & $0.46-4.22$ & 0.56 \\
$35-44$ & $156(22.4)$ & 1.22 & $0.41-4.00$ & 0.68 \\
$45-54$ & $70(10.0)$ & 0.64 & $0.17-2.39$ & 0.50 \\
$55-64$ & $42(6.0)$ & 0.44 & $0.09-2.15$ & 0.31 \\
$>$ 64 & $37(5.3)$ & 0.33 & $0.05-1.94$ & 0.22 \\
Total & $\mathbf{6 9 5 ( 1 0 0 )}$ & $\mathbf{0 . 9 6}$ & $\mathbf{0 . 6 4 - 1 . 4 2}$ & $\mathbf{0 . 8 8}$ \\
\hline
\end{tabular}

$\overline{\mathrm{OR}}=$ odds ratio, $\mathrm{CI}=$ confidence interval, $P$-value $=$ level of marginal significance

\section{NTM-HIV co-infection}

Males constituted $66.7 \%$ and females $33.3 \%$ of NTM cases. Seven $(46.7 \%)$ of the NTM cases were co-infected with HIV, five $(33.3 \%)$ were HIV negative, and three $(20 \%)$, all of them males, were of unknown HIV status (table 4). Three of the NTM-HIV co-infection cases were on antiretroviral therapy.

Table 4: NTM-HIV co-infection

\begin{tabular}{lllr}
\hline NTM and HIV status & Males (\%) & Females (\%) & Total (\%) \\
\hline NTM with HIV infection & $4(26.7)$ & $3(20)$ & $7(46.7)$ \\
NTM without HIV & $3(20)$ & $2(13.3)$ & $5(33.3)$ \\
NTM with HIV status unknown & $3(20)$ & 0 & $3(20)$ \\
Total & $\mathbf{1 0 ( 6 6 . 7 )}$ & $\mathbf{5 ( 3 3 . 3 )}$ & $\mathbf{1 5 ( 1 0 0 )}$ \\
\hline
\end{tabular}

\section{Discussion}

Tuberculosis is a serious opportunistic infection among people living with HIV/AIDS (PLWHA) worldwide, whereas HIV co-infection modifies the natural history and clinical presentation, and adversely affects the outcome of $\mathrm{TB}^{24}$. The increasing $\mathrm{TB}$ burden in Kenya has also been attributed to the concurrent HIV/AIDS epidemic, with the 2007, 2008 and 2009 Division of Leprosy, Tuberculosis and Lung Disease (DLTLD) annual reports ${ }^{16,25,26}$ indicating persistently high rates (44-48\%) of TB-
HIV co-infection. The current study however, reports a slightly lower $(42.7 \%)$ co-infection rate. The difference though not significant could be due to the study participants being from the rural population where there is less crowding thereby reducing the chances of mycobacteria infection transmission. However, both DLTLD and the present study report much higher TB-HIV co-infection rates than the global rate of $14.8 \%$ among the 9.27 million new cases of TB that occurred in $2007{ }^{27}$. 
In Kenya, like elsewhere, HIV has significantly influenced the increased proportion of smear negative TB since 2005, which has contributed to increased TB morbidity and mortality, mostly affecting male ${ }^{16}$. In the current study, $5.8 \%$ the $\mathrm{TB}$ cases were $\mathrm{ZN}$ smear negative. The present study suggests that HIV co-infection with does not seem to alter the prevalence of TB among males and females. This finding is consistent with the DLTLD annual reports ${ }^{16,25,26}$. However, it does significantly contribute to TB patients requiring re-treatment ${ }^{16}$. The DLTLD annual reports $16,25,26$ and results of the present study demonstrate that TB-HIV co-infection rate is still pervasive in Kenya as the rest of sub-Saharan Africa. This presents serious challenges in many developing countries ?.

The HIV co-infection makes diagnosis and management of TB in children more difficult because other HIV-related diseases, such as lymphocytic interstitial pneumonitis, may present in a similar way to PTB or miliary $\mathrm{TB}^{28}$. Apart from being less readily available poor resource settings, interpretation of tuberculin skin testing and chest X-ray is less reliable, and when TB-HIV co-infection is common in adults a positive contact history is less specific if the contact is the child's guardian. The child is at risk of transmission of either or both diseases. Moreover children with TB and advanced HIV disease may not respond as well to TB treatment ${ }^{28-30}$. Testing for HIV infection can be helpful, especially if the result is negative, as it increases the likelihood of a diagnosis of TB. However, a positive HIV result clearly does not exclude the possibility of $\mathrm{TB}^{31}$.

Severe disseminated TB in HIV/AIDS cases is well-recognized but early disease may be characterized by few or no symptoms. The HIV/AIDS compromises the sensitivity of immunodiagnostic methods to ascertain latent $\mathrm{TB}^{24}$. However, chemoprevention of HIV/AIDS associated TB is often effective, albeit benefits restricted to those with evidence of immune sensitization and appear shortlived in areas of high TB burden. Although promising advances in the microbiological diagnosis of TB have recently occurred, the diagnosis of HIV/AIDS associated TB remains difficult because of more frequent presentation as sputum negative or extrapulmonary disease ${ }^{24,32}$. Moreover, the level of sophistication and cost associated with these new and more sensitive TB diagnosis techniques have made their general applicability unfeasible in developing countries ${ }^{33}$.

Lack or poor coordination between the TB and the HIV/AIDS programmes has been reported to frustrate TB control efforts. It has been observed that once a patient is diagnosed with TB, they are sent to a TB clinic with little further interaction with the HIV/AIDS specialists even in the same health facility ${ }^{5}$. However, the problem faced by high burden countries has spurred the drive to tackle the dual epidemic with coordinated response intended to draw on the strength of the directly observed therapy short course (DOTS) strategy. In Kenya for instance, the government has set up mechanisms of collaboration between TB and HIV/AIDS national programmes whose activities target the reduction of HIV burden amongst TB patients and reduction of TB burden amongst People living with HIV/AIDS ${ }^{25}$. Strengthening the DOTS strategy seems to be an essential strategy in this case, but not sufficient by itself ${ }^{34}$. Additional existing interventions to prevent TB among HIV/AIDS patients such as prophylaxis and intensified case finding are necessary to implement ${ }^{5}, 34$. In our study finding, there was relatively low, although statistically insignificant prevalence of TB - HIV co-infections among paediatric participants implies that infection prevention strategies should target this age group.

Management of TB among HIV/AIDS patients is even more problematic and complex ${ }^{24}$, requiring highly skilled personnel to administer medication, in order to avoid overlapping drug toxicities and adverse interactions like reduction of the efficacy of the drugs $\mathrm{s}^{24}$. Nevertheless, consensus is developing that antiretroviral therapy should be provided as soon as practicable after starting TB treatment in HIV- coinfected persons. However, this has the consequence of increasing the frequency of immune reconstitution inflammatory syndrome (IRIS), the pathogenesis and management of which is currently poorly defined ${ }^{24}$. In Kenya TB in many healthcare facilities is managed by clinical officers (paramedical practitioners) who lack the necessary skills for specialised management of TBHIV/AIDS patients, the adoption of the WHO ${ }^{35} \mathrm{~TB}$ management guidelines by the government notwithstanding.

In Kenya, TB culture is not routinely done, and treatment of new cases is based on $\mathrm{ZN}$ smear microscopy, clinical symptoms, and occasionally augmented with chest X-ray in some health facilities. However, not all acid fast bacilli represent mycobacteria, let alone $M$. tuberculosis complex. Nontuberculous mycobacteria (NTM) ${ }^{36}$ and some other bacterial species including Nocardia species which are widely spread in the environment yield positive results in $\mathrm{ZN}$ smear detection of acid-fast bacilli (AFB) and may present with similar radiological features ${ }^{37}$. Some of the $46.4 \% \mathrm{ZN}$ smear positive but culture negative cases treated as TB in the current study could have been NTM or other acid-fast bacterial diseases misdiagnosed as TB. There is also concern about $6.4 \%$ 
of cases who were smear negative but culture positive implying that such cases do not get appropriate diagnosis and treatment, taking into consideration that smear microscopy is widely used in most of our health care facilities. From the foregoing and the results of current study, it is evident that TB treatment in Kenya is not fully evidence-based. The increasing cases of HIV/AIDS associated NTM mycobacterioses ${ }^{9}$ could be misdiagnosed as TB and put on anti-TB chemotherapy, even though the treatment of NTM disease is generally not directly analogous to TB treatment ${ }^{32,38}$. Multi-drug regimens are used for NTM disease treatment, the cornerstone agents being a newer macrolide (azithromycin, clarithromycin) ${ }^{38}$, ethambutol, and rifamycin, and require prolonged durations of therapy aimed to facilitate clearance of the mycobacteria and minimize the emergence of drug resistance ${ }^{32,38}$.

\section{Conclusion}

The TB-HIV/AIDS co-infection rate was persistently high in Kenya, an indication that the TB-HIV/AIDS dual epidemic will continue to portend a huge challenge to Kenya for many years to come.

\section{Recommendations}

The persistently high TB-HIV/AIDS co-infection rate calls for the intensification and strengthening of the collaborative activities between the TB and HIV/AIDS National programmes and improved diagnostic services. This includes the need to improve TB management skills among clinical and laboratory personnel involved in taking care of TB patients at district and lower level health facilities where the majority of the population seek healthcare. Furthermore, the pervasiveness of TB and HIV/ AIDS in western Kenya also underscores the need for more efforts and resources to increase knowledge and access to TB and HIV/AIDS healthcare services, with emphasis on tackling cultural, socio-economic, political, and biological factors that make young people disproportionately vulnerable to TB-HIV/AIDS, and addressing the obstacles to universal access to ART.

\section{Competing interests}

The authors declare that they have no competing interests.

\section{Acknowledgement}

We thank the Medical Officers of Health, Medical Superintendents, District Leprosy and Tuberculosis Coordinators, Laboratory staff and clinical and nursing staff at Narok, Kericho, Lodwar, Uasin Gishu, Bungoma, Busia, Kisumu, Migori, District Hospitals, Kisii Level 5 Hospital and Nakuru Provincial General Hospital who greatly us assisted with specimen and data collection for this study. We are also indebted to the Laboratory Technicians at the Mycobacteria Reference Laboratory, MUSOM who assisted with laboratory work. We wish to thank the Global Fund TB Round 5 Project for funding this study through the Government of Kenya.

\section{References}

1. WHO Report. Global tuberculosis control: Surveillance, Planning, Financing, Communicable Diseases. WHO, Geneva, 2002.

2. UNAIDS. Global Facts and Figures: The global AIDS epidemic. 2009.

3. WHO. Tuberculosis Facts, 2009. www.who.int/ $\mathrm{t}$ b / p u b l i c a t i o n s/ $2009 /$ tbfactsheet_2009_one_page.pdf

4. Bakare N, Miller V. HIV-TB co-infection: meeting the challenge. Report of the Forum for Collaborative HIV Research and TB/HIV. Working Group of the Stop TB Partnership Symposium and Roundtable discussions on HIV / TB. July 22-23, 2007, Sydney Australia, report date: November 2, 2007.

5. Nullis-Kapp C. Africa is worst hit by dual epidemic. Bull World Health Organ 2005; 83(3): 165-6.

6. Nunn P, Brindle R, Carpenter L, Odhiambo J, Wasunna K, Newnham R, et al. Cohort-study of human immunodeficiency virus infection in patients with tuberculosis in Nairobi, Kenya. Analysis of early (6 months) mortality. Am Rev Respir Dis. 1992; 146: 849-54.

7. Daley CL, Small PM, Scheeter GF, Schoolink GK, McAda, RA, Jacobs WR at al. An outbreak of tuberculosis with accelerated progression among persons infected with the Human immunodeficiency virus. An analysis using restriction - fragment -length polymorphism. $N$. Eng J. Med 1992; 262: 231.

8. Villarino ME, Dooley SW, Geither LJ, Castro K, Snider D. Management of persons exposed to multidrug-resistant tuberculosis. MMWR Morb Mortal Wkly Rep. 1992; 41 (RR-11): 61-71.

9. Mallory KF, Churchyard GJ, Kleinschmidt I, Decock KM, Corbett EL. The Impact of HIV Infection on Recurrence of Tuberculosis in South African Gold Miners. Int J Tuberc Lung Dis 2000; 4(5): 455-62. 
10. Wallis RS, Vjecha M, Nelson M, Okwera A, Byekwaso F, Nyole S, Kabengera S, et al. Influence of tuberculosis on human $\beta 2$-immunodeficiency virus (HIV-1): Enhanced cytokine expression and elevated microglobulin in HIV-1-associated tuberculosis. J Infect Dis 1993; 167:43-8.

11. Ackah AN, Caulibaly D, Digbeu H, Diallo K, Vetter KM, Coulibaly IM, Greenberg AE, et al. Response to treatment, mortality, and CD4 lymphocyte counts in HIV-infected persons with tuberculosis in Abidjan, Ivory Coast. Lancet 1995; 354(8950):607-610.

12. Whalen C, Lahart C, Hom D, Lahart C, Simberkoff M, Ellner J. Accelerated course of HIV infection after tuberculosis. Am J Respir Crit Care Med 1995; 151:129-35.

13. Grant DA, Sidile K, Domousa K, Bonard D, SyllaKoko F, Dosso M., et al. Spectrum of disease among HIV-infected adults hospitalized in a respiratory medicine unit in Abidjan, Cote d'Ivoire. Int J Lung Dis 1998; 2(11): 926-34.

14. WHO. AIDS, Global situation of the HIV/AIDS pandemic. Wkly Epidemiol Rec 1995; 70: 193.

15. Global Health Council. Co-infection. http:// 72.3.236.96/infectious_diseases/co_infection/

16. Division of Leprosy, tuberculosis and Lung Disease (DLTLD), Ministry of Public Health and Sanitation, Government of Kenya. Annual Report, 2009.

17. Azevedo MJ, Prater GS, Hayes SC. Human immunodeficiency virus and tuberculosis coinfection in Kenya: Environment, Resources and Culture. International Journal of sociology and Anthropology. 2010; 2(4): 55-65.

18. Johnson, JL, Ellner, JJ. Tuberculosis and Atypical Mycobacterial Infections. In: Tropical Infectious Diseases: Principles, Pathogens \& Practice, Vol. 1. Guerrrant, R. L., Walker, D. H. and Weller, P. F. eds. Churchill Livingstone, Philadelphia. 1999. Pp. 443-73.

19. Wolinsky E. Mycobacterial diseases other than tuberculosis. Clin Infect Dis. 1992; 15:(1): 1-10.

20. National Leprosy and Tuberculosis Programme (NLTP). (2003), Ministry of Health, Republic of Kenya. Tuberculosis Guidelines.

21. World Health Organization (WHO). Guidelines for the prevention of tuberculosis in healthcare facilities in resource-limited settings. WHO, Geneva. 1999.

22. Ebersole, L. L. Acid-fast stain procedures. In: Isenberg HD, ed. Clinical Micribiology Procedure Handbook. Washington, DC: ASM Press; 1992:3.5.1-3.5.10.

23. Find. MGIT ${ }^{\mathrm{TM}}$ Procedure Manual for BACTEC TM MGIT 960M TB System, 2006.
24. Schutz, C., Meinjtjes, G., Almajid, F., Wilkinson, R. J. and Pozniak, A. Clinical management of tuberculosis and HIV-1 co-infection. ERJ 2010; 36 (6): 1460-1481.

25. Division of Leprosy, tuberculosis and Lung Disease (DLTLD), Ministry of Public Health and Sanitation, Government of Kenya. Annual Report, 2007.

26. Division of Leprosy, tuberculosis and Lung Disease (DLTLD), Ministry of Public Health and Sanitation, Government of Kenya. Annual Report, 2008.

27. WHO Report. Global tuberculosis control: Surveillance, Planning, Financing. Geneva, 2007.

28. National institute of child health and human development (NICHD). (2007). http:// www.nichd.nih.gov

29. National institute of health (NIH) (2007). http:/ /www.nih.gov

30. National institute of mental health (NIMH). (2007). http://www.nimh.nih.gov

31. World Health Organization. Treatment of tuberculosis. Guidelines for National Programmes. WHO/CDS/TB 2003: 313). Geneva. 2003.

32. American Thoracic Society (ATS). An Official ATS/IDSA Statement: Diagnosis, Treatment, and Prevention of Nontuberculous Mycobacterial Diseases. 2007.

33. Buijtels PCAM. Clinical relevance of nontuberculous mycobacteria in Zambia. PhD thesis. 2007

34. World Health Organization. Interim policy on collaborative TB/HIV activities. Geneva. 2005.

35. World Health Organization WHO). Treatment of Tuberculosis: guidelines for national programmes 4th edition, WHO/HTM/TB/2009.420. 2009.

36. Buijtels PCAM, van der Sande MAB, de Graaff CS, Parkinson S, Verbrugh HA, Petit PLC, et al. Nontuberculous mycobacteria, Zambia. Emerg Infect Dis 2009; 15(2): 242-249.

37. Olson ES, Simpson AJH, Norton AJ and Das SS. Not everything acid fast is Mycobacterium tuberculosis: a case report. J Clin Pathol 1998; 51: 535-536.

38. Griffith DE, Aksamit T, Brown-Elliot BA, Catanzaro A, Daley C, Gordin F, et al. ATS Mycobacterial Diseases Subcommittee; American Thoracic Society; Infectious Diseases Society of America. An official ATS/IDSA statement: diagnosis, treatment, and prevention of nontuberculous mycobacterial diseases. Am. J. Respir. Crit Care Med 2007; 175: 367-416. 